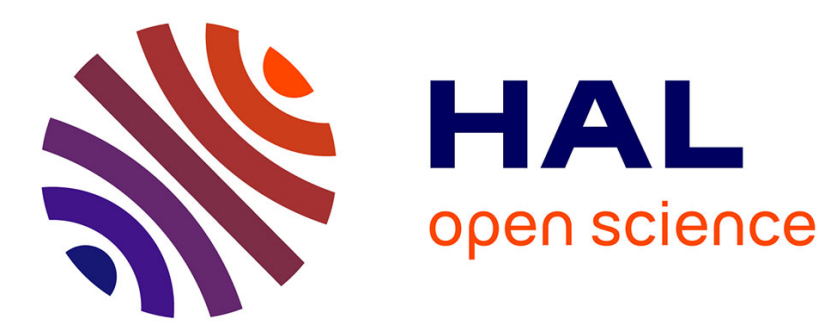

\title{
Adaptive wavelet estimation of a density from mixtures under multiplicative censoring
}

Yogendra P. Chaubey, Christophe Chesneau, Hassan Doosti

\section{To cite this version:}

Yogendra P. Chaubey, Christophe Chesneau, Hassan Doosti. Adaptive wavelet estimation of a density from mixtures under multiplicative censoring. 2013. hal-00918069

\section{HAL Id: hal-00918069 \\ https://hal.science/hal-00918069}

Preprint submitted on 12 Dec 2013

HAL is a multi-disciplinary open access archive for the deposit and dissemination of scientific research documents, whether they are published or not. The documents may come from teaching and research institutions in France or abroad, or from public or private research centers.
L'archive ouverte pluridisciplinaire HAL, est destinée au dépôt et à la diffusion de documents scientifiques de niveau recherche, publiés ou non, émanant des établissements d'enseignement et de recherche français ou étrangers, des laboratoires publics ou privés. 
arXiv: math.PR/0000000

\title{
Adaptive wavelet estimation of a density from mixtures under multiplicative censoring
}

\author{
Yogendra P. Chaubey ${ }^{1, *}$ Christophe Chesneau ${ }^{2, \dagger}$ \\ and \\ Hassan Doosti ${ }^{3}$ \\ ${ }^{1}$ Department of Mathematics and Statistics, Concordia University, Montréal, QC H3G \\ 1M8, CANADA. e-mail: chaubey@alcor.concordia.ca \\ ${ }^{2}$ Laboratoire de Mathématiques Nicolas Oresme, Université de Caen BP 5186, F 14032 \\ Caen Cedex, FRANCE. e-mail: chesneau@math.unicaen.fr \\ ${ }^{3}$ Department of Mathematics, Kharazmi University, Tehran, IRAN and Department of \\ Mathematics and Statistics, The University of Melbourne, Melbourne, AUSTRALIA. \\ e-mail: h.doosti@tmu.ac.ir - hassan.doosti@unimelb.edu.au
}

\begin{abstract}
In this paper, a mixture model under multiplicative censoring is considered. We investigate the estimation of a component of the mixture (a density) from the observations. A new adaptive estimator based on wavelets and a hard thresholding rule is constructed for this problem. Under mild assumptions on the model, we study its asymptotic properties by determining an upper bound of the mean integrated squared error over a wide range of Besov balls. We prove that the obtained upper bound is sharp.
\end{abstract}

AMS 2000 subject classifications: Primary 62G07, 62G20; secondary $60 \mathrm{~K} 35$.

Keywords and phrases: Density estimation, mixture, Besov balls, wavelets, hard thresholding.

\section{Introduction}

First of all, let us present the standard density estimation problem under multiplicative censoring as described in Vardi (1989). We observe $n$ independent random variables $Y_{1}, \ldots, Y_{n}$ where

$$
Y_{i}=U_{i} X_{i}, \quad i \in\{1, \ldots, n\},
$$

$U_{1}, \ldots, U_{n}$ are $n$ unobserved i.i.d. random variables having the uniform distribution on $[0,1]$ and $X_{1}, \ldots, X_{n}$ are $n$ unobserved i.i.d. random variables. We suppose that $X_{1}, \ldots, X_{n}$ and $U_{1}, \ldots, U_{n}$ are independent. We aim to estimate $f$, the unknown density of $X_{1}$, from $Y_{1}, \ldots, Y_{n}$.

It is shown in Vardi (1989) [see also Vardi and Zhang (1992)] how this model unifies several well-studied statistical problems, including non-para-

\footnotetext{
*Supported in part by a Discovery Grant from NSERC of Canada.

†Supported in part by ANR grant NatImages, ANR-08-EMER-009. 
metric inference for renewal processes, certain non-parametric deconvolution problems and estimation of decreasing densities. Among the methods used to solve this problem, there is the method of singular value decomposition (or orthonormal series based expansion) developed in Andersen and Hansen (2001), the method using the wavelet technique used in Abbaszadeh et al. (2012) and the kernel method developed in Asgharian et al. (2012).

In this paper, we consider a more general model incorporating mixtures. Here, we observe $n$ independent random variables $Y_{1}, \ldots, Y_{n}$ according to (1.1) where $U_{1}, \ldots, U_{n}$ are unobserved i.i.d. random variables having the uniform distribution on $[0,1]$ and $X_{1}, \ldots, X_{n}$ are unobserved independent random variables but not (necessarily) identically distributed: for any $i \in\{1, \ldots, n\}, X_{i}$ depends on an unobserved random indicator $I_{i}$ taking its values in $\{1, \ldots, m\}$. Applying the Bayes theorem, the density of $X_{i}$ is the following finite mixture:

$$
h_{i}(x)=\sum_{d=1}^{m} w_{d}(i) f_{d}(x)
$$

where $w_{d}(i)=\mathbb{P}\left(I_{i}=d\right)$ and $f_{d}$ is the conditional density of $X_{i}$ given $\left\{I_{i}=d\right\}$. We suppose that all these densities are unknown, the weight $w_{d}(i)$ is known, and $X_{1}, \ldots, X_{n}$ and $U_{1}, \ldots, U_{n}$ are independent. For a fixed $\nu \in\{1, \ldots, m\}$, we aim to estimate $f_{\nu}$ i.e. the $\nu$-th component of the mixture, from $Y_{1}, \ldots, Y_{n}$.

Note that, when $m=1, w_{1}(1)=\ldots=w_{1}(n)=1$ and $f_{\nu}=f_{1}$, we arrive at the density estimation problem under multiplicative censoring described in the first paragraph. The problem of estimating $f_{\nu}$ from $X_{1}, \ldots, X_{n}$ (not $\left.Y_{1}, \ldots, Y_{n}\right)$ has been considered in several papers [see e.g. Maiboroda (1996), Hall and Zhou (2003), Pokhyl'ko (2005) and Prakasa Rao (2010)]. However, to the best of our knowledge, the estimation of $f_{\nu}$ from $Y_{1}, \ldots, Y_{n}$ is a new statistical problem that has potential applications in signal processing, biology, industry and telecommunications. For instance, consider the example of a sensor network (i.e. a collection of spatially distributed autonomous sensors intended to measure physical phenomena at diverse locations). Suppose that it contains $m$ nodes, each sensor records only one physical value of the phenomena and only one sensor information is collected at a time. Now, given $n$ multiplicative noisy versions $Y_{1}, \ldots, Y_{n}$ of the physical phenomena from non-necessarily identified sensors (i.e. unobserved $I_{1}, \ldots, I_{n}$ ), the goal is to estimate the density function of the physical phenomena at any sensor $\nu \in\{1, \ldots, m\}$. An overview of sensor networks can be found in Akyildiz et al. (2002). The reader may be referred to Chabert et al. (2004) and references therein for applications in multiplicative noise set-up in signal processing.

Among the numerous existing methods (such as Kernel, Spline, Wavelets and others) [see e.g. Prakasa Rao $(1983,1999)$ and Tsybakov (2004)], we investigate the estimation of $f_{\nu}$ via wavelet methods. They are attractive for nonparametric function estimation because of their spatial adaptivity, computational efficiency and asymptotic optimality properties. They can achieve near optimal convergence rates over a wide range of function classes (such as Besov balls) and enjoy excellent mean integrated squared error (MISE) properties when used to esti-

imsart-generic ver. 2009/12/15 file: bj4_yogen_rev.tex date: December 12, 2013 
mate spatially inhomogeneous function [see Antoniadis (1997) and Härdle et al. (1998)].

In this paper, we develop an adaptive wavelet estimator for $f_{\nu}$ based on the hard thresholding rule [introduced in Donoho and Johnstone (1994, 1995)]. In its construction, we use the new methodology developed in Abbaszadeh et al. (2012) for the density estimation under bias and multiplicative censoring, the "observations thresholding technique" introduced by Delyon and Juditsky (1996), and some tools on mixtures described in Maiboroda (1996). We evaluate the performance of the proposed density estimator by considering the MISE and assuming that $f_{\nu}$ belongs to a Besov ball. Under mild assumptions on the weights of the mixtures, we prove that our estimator attains a sharp rate of convergence: it is the one attained by the "best" non-adaptive linear wavelet estimator up to a logarithmic term, where "best" is used in the sense of minimum MISE.

The outline of the paper is as follows. We provide detailed assumptions on the mixture model considered here in Section 2. Section 3 describes the wavelet basis on $[0,1]$ and the Besov balls. The estimators are presented in Section 4 and the main results are provided in Section 5. The proofs are gathered in Section 6.

\section{Assumptions}

Additional assumptions on our model are presented below.

- Without loss of generality, we assume that the support of $f_{d}$ is $[0,1]$ and $f_{d} \in \mathbb{L}^{2}([0,1])$ for any $d \in\{1, \ldots, m\}$, where, for any $p \geq 1$,

$$
\mathbb{L}^{p}([0,1])=\left\{u:[0,1] \rightarrow \mathbb{R} ;\|u\|_{p}=\left(\int_{0}^{1}|u(x)|^{p} d x\right)^{1 / p}<\infty\right\} .
$$

Note that, for any $i \in\{1, \ldots, n\}$, the density of $Y_{i}$ is

$$
g_{i}(x)=\int_{x}^{1} \frac{h_{i}(y)}{y} d y, \quad x \in[0,1],
$$

where $h_{i}$ is as given in (1.2).

We suppose that there exists a known constant $C_{*}>0$ such that

$$
\max _{i \in\{1, \ldots, n\}} \sup _{x \in[0,1]} g_{i}(x) \leq C_{*} .
$$

Such a boundedness assumption is standard in the density estimation problems from mixtures [see e.g. Maiboroda (1996), Pokhyl'ko (2005) and Prakasa Rao (2010)].

- We now formulate some assumptions on the weights of the mixture. Consider the matrix

$$
\Gamma_{n}=\left(\frac{1}{n} \sum_{i=1}^{n} w_{k}(i) w_{\ell}(i)\right)_{(k, \ell) \in\{1, \ldots, m\}^{2}} .
$$

imsart-generic ver. 2009/12/15 file: bj4_yogen_rev.tex date: December 12, 2013 
We assume that $\operatorname{det}\left(\Gamma_{n}\right)>0$.

Then the solution of the following quadratic objective with linear constraints,

$$
\min _{b \in \mathbb{R}^{n}} \frac{1}{n} \sum_{i=1}^{n} b_{i}^{2} \text { such that } \frac{1}{n} \sum_{i=1}^{n} b_{i} w_{d}(i)=\delta_{\nu, d}, \text { for } d \in\{1, \cdots, m\},
$$

where $\delta_{\nu, d}$ denotes the Kronecker delta, is unique and is denoted by $\left(a_{\nu}(1), \ldots, a_{\nu}(n)\right)$. Specifically, we have

$$
a_{\nu}(i)=\frac{1}{\operatorname{det}\left(\Gamma_{n}\right)} \sum_{k=1}^{m}(-1)^{k+\nu} \zeta_{\nu, k}^{n} w_{k}(i)
$$

where $\zeta_{\nu, k}^{n}$ denotes the determinant of the minor $(\nu, k)$ of the matrix $\Gamma_{n}$. Details concerning the above results can be found in Maiboroda (1996) and Pokhyl'ko (2005).

Remark 2.1. The definition of $\left(a_{\nu}(1), \ldots, a_{\nu}(n)\right)(2.3)$ allows us to

- construct unbiased wavelet coefficients estimators well adapted to the structure of our mixture model (see Proposition 4.1),

- obtain sharp rate of convergence (the quantity $(1 / n) \sum_{i=1}^{n} a_{\nu}^{2}(i)$ will play a central role); see Theorems 5.1 and 5.2.

We set

$$
\rho_{n}=\frac{1}{n} \sum_{i=1}^{n} a_{\nu}^{2}(i)
$$

and, for technical reasons, we suppose that $\lim _{n \rightarrow \infty} n / \rho_{n}=\infty$.

\section{Wavelets and Besov balls}

\subsection{Wavelets}

For the purposes of this paper, we use the compactly supported wavelet basis on $[0,1]$ briefly described below.

Let $N \geq 10$ and $\phi$ and $\psi$ be the initial wavelet functions of the Daubechies wavelets $d b 2 N$. In particular, these functions are compactly supported with $\operatorname{supp}(\phi) \subseteq[1-N, N]$ and $\operatorname{supp}(\psi) \subseteq[1-N, N]$ (for the sake of simplicity), and belong to $\mathcal{C}^{1}$.

We define the functions $\phi_{j, k}$ and $\psi_{j, k}$ by dilation and translation of $\phi$ and $\psi$ respectively, and normalization:

$$
\phi_{j, k}(x)=2^{j / 2} \phi\left(2^{j} x-k\right), \quad \psi_{j, k}(x)=2^{j / 2} \psi\left(2^{j} x-k\right) .
$$

Then, with an appropriate treatment at the boundaries, there exists an integer $\tau$ such that, for any integer $\ell \geq \tau$,

$\mathcal{B}=\left\{\phi_{\ell, k}, k \in\left\{0, \ldots, 2^{\ell}-1\right\} ; \psi_{j, k} ; j \in \mathbb{N}-\{0, \ldots, \ell-1\}, k \in\left\{0, \ldots, 2^{j}-1\right\}\right\}$ 
is an orthonormal basis of $\mathbb{L}^{2}([0,1])$.

For any integer $\ell \geq \tau$, a function $u \in \mathbb{L}^{2}([0,1])$ can be expanded on $\mathcal{B}$ as

$$
u(x)=\sum_{k=0}^{2^{\ell}-1} \alpha_{\ell, k} \phi_{\ell, k}(x)+\sum_{j=\ell}^{\infty} \sum_{k=0}^{2^{j}-1} \beta_{j, k} \psi_{j, k}(x), \quad x \in[0,1],
$$

where $\alpha_{j, k}$ and $\beta_{j, k}$ are the wavelet coefficients of $u$ defined by

$$
\alpha_{j, k}=\int_{0}^{1} u(x) \phi_{j, k}(x) d x, \quad \beta_{j, k}=\int_{0}^{1} u(x) \psi_{j, k}(x) d x .
$$

We refer to Cohen et al. (1993) and Mallat (2009) for more details.

\subsection{Besov balls}

As is traditional in the wavelet estimation literature, we will investigate the performances of our estimators by assuming that the unknown function $f_{\nu}$ belongs to a Besov ball defined below.

Let $M>0, s>0, p \geq 1$ and $r \geq 1$. Set, for every measurable function $u$ on $[0,1]$ and $\epsilon \geq 0$,

$$
\Delta_{\epsilon}(u)(x)=u(x+\epsilon)-u(x),
$$

$\Delta_{\epsilon}^{2}(u)(x)=\Delta_{\epsilon}\left(\Delta_{\epsilon}(u)\right)(x)$ and identically, $\Delta_{\epsilon}^{N}(u)(x)=\Delta_{\epsilon}^{N-1}\left(\Delta_{\epsilon}(u)\right)(x)$ for any positive integer $N$. Let

$$
\rho^{N}(t, u, p)=\sup _{\epsilon \in[-t, t]}\left\|\Delta_{\epsilon}^{N}(u)\right\|_{p}
$$

Then, for $s \in[0, N)$, we define the Besov ball $B_{p, r}^{s}(M)$ of radius $M>0$ by

$$
B_{p, r}^{s}(M)=\left\{u \in \mathbb{L}^{p}([0,1]) ;\left(\int_{0}^{1}\left(\frac{\rho^{N}(t, u, p)}{t^{s}}\right)^{r} \frac{d t}{t}\right)^{1 / r} \leq M\right\},
$$

with the usual modifications if $p=\infty$ or $r=\infty$.

The parameter $s$ is related to the smoothness of the function $u$, whereas $p$ and $r$ are norm parameters.

We have the following equivalence (see Härdle et al. (1998), Corollary 9.1): $u \in B_{p, r}^{s}(M)$ with $s \in(0, N)$ if and only if there exists a constant $M^{*}>0$ (depending on $M$ ) such that the associated wavelet coefficients given by (3.1) satisfy

$$
\begin{aligned}
& 2^{\tau(1 / 2-1 / p)}\left(\sum_{k=0}^{2^{\tau}-1}\left|\alpha_{\tau, k}\right|^{p}\right)^{1 / p}+\left(\sum_{j=\tau}^{\infty}\left(2^{j(s+1 / 2-1 / p)}\left(\sum_{k=0}^{2^{j}-1}\left|\beta_{j, k}\right|^{p}\right)^{1 / p}\right)^{r}\right)^{1 / r} \\
& \leq M^{*}
\end{aligned}
$$

with the usual modifications if $p=\infty$ or $r=\infty$. For a particular choice of $s, p$ and $r, B_{p, r}^{s}(M)$ contains the Hölder and Sobolev balls [see Meyer (1990)]. 


\section{Estimators}

\subsection{Wavelet coefficients estimators}

The first step in estimating $f_{\nu}$ consists of expanding $f_{\nu}$ on $\mathcal{B}$ and estimating its unknown wavelet coefficients. Here we adapt the methodology of Abbaszadeh et al. (2012). We consider the operator $T: \mathcal{C}^{1}([0,1]) \rightarrow \mathcal{C}^{0}([0,1])$ defined by

$$
T(u)(x)=(x u(x))^{\prime}=u(x)+x u^{\prime}(x), \quad u \in \mathcal{C}^{1}([0,1]), \quad x \in[0,1] .
$$

For any integer $j \geq \tau$ and any $k \in\left\{0, \ldots, 2^{j}-1\right\}$, we estimate the wavelet coefficients of $f_{\nu}$, i.e. $\alpha_{j, k}=\int_{0}^{1} f_{\nu}(x) \phi_{j, k}(x) d x$ and $\beta_{j, k}=\int_{0}^{1} f_{\nu}(x) \psi_{j, k}(x) d x$, by respectively

$$
\widehat{\alpha}_{j, k}=\frac{1}{n} \sum_{i=1}^{n} a_{\nu}(i) T\left(\phi_{j, k}\right)\left(Y_{i}\right)
$$

and

$$
\widehat{\beta}_{j, k}=\frac{1}{n} \sum_{i=1}^{n} a_{\nu}(i) T\left(\psi_{j, k}\right)\left(Y_{i}\right) \mathbf{1}_{\left\{\left|a_{\nu}(i) T\left(\psi_{j, k}\right)\left(Y_{i}\right)\right| \leq \eta_{j}\right\}},
$$

where $Y_{i}$ is defined by (1.1), $a_{\nu}(i)$ by $(2.4), T$ by (4.1), $\mathbf{1}_{\mathcal{A}}$ is the indicator function on $\mathcal{A}$ for any random event $\mathcal{A}$,

$$
\begin{gathered}
\eta_{j}=\theta 2^{j} \sqrt{\frac{n \rho_{n}}{\ln \left(n / \rho_{n}\right)}}, \\
\theta=\sqrt{2 C_{*}\left(1+\int_{1-N}^{N}\left(\psi^{\prime}(y)\right)^{2} d y\right)},
\end{gathered}
$$

$C_{*}$ is the constant in (2.1) and $\rho_{n}$ is defined by (2.5).

The wavelet coefficient estimators $\widehat{\alpha}_{j, k}$ and $\widehat{\beta}_{j, k}$ are motivated by the properties of the constants $a_{\nu}(i)$ defined in (2.3) and (2.4). We can write

$$
f_{\nu}(x)=\frac{1}{n} \sum_{i=1}^{n} a_{\nu}(i) h_{i}(x)
$$

and use the fact that (see the details in the proof of Proposition 4.1)

$$
\int_{0}^{1} h_{i}(x) \phi_{j, k}(x) d x=\mathbb{E}\left(T\left(\phi_{j, k}\right)\left(Y_{i}\right)\right) .
$$

Thus $\widehat{\alpha}_{j, k}$ provides an unbiased estimator of $\alpha_{j, k}$ where as $\widehat{\beta}_{j, k}$ is based on the "observations thresholding technique" that had been firstly introduced in Delyon and Juditsky (1996) to estimate the regression function in a regression 
model with general errors. In our study, the role of its selection is to provide a good estimator of $\beta_{j, k}$ under mild assumptions on the distributions of $I_{1}, \ldots, I_{n}$. To be more specific, we have no boundedness assumptions on the eigenvalues of the matrix $\Gamma_{n}$ (as in Autin and Pouet (2011)) and, more generally, on $a_{\nu}(i)$. This point is developed in Remarks 5.3 and 5.4. Our statistical approach has been used for other mixture models in Chesneau (2011a,b) and Chesneau and Fadili (2012).

Proposition 4.1. For any integer $j \geq \tau$ and any $k \in\left\{0, \ldots, 2^{j}-1\right\}$, let $\alpha_{j, k}$ and $\beta_{j, k}$ be the wavelet coefficients of $f_{\nu}$ as given in (3.1). Then

- $\widehat{\alpha}_{j, k}$ defined by $(4.2)$ is an unbiased estimator of $\alpha_{j, k}$,

- we have

$$
\mathbb{E}\left(\frac{1}{n} \sum_{i=1}^{n} a_{\nu}(i) T\left(\psi_{j, k}\right)\left(Y_{i}\right)\right)=\beta_{j, k} .
$$

Remark 4.1. In addition to the unbiased properties described in Proposition $4.1, \widehat{\alpha}_{j, k}$ and $\widehat{\beta}_{j, k}$ satisfy powerful moments and concentration inequalities, i.e., Moments inequality: there exists a constant $C>0$ such that

$$
\mathbb{E}\left(\left(\widehat{\alpha}_{j, k}-\alpha_{j, k}\right)^{2}\right) \leq C 2^{2 j} \frac{\rho_{n}}{n}
$$

and

$$
\mathbb{E}\left(\left(\widehat{\beta}_{j, k}-\beta_{j, k}\right)^{4}\right) \leq C 2^{4 j} \frac{\left(\rho_{n} \ln \left(n / \rho_{n}\right)\right)^{2}}{n^{2}} .
$$

Concentration inequality: for a large enough $\kappa$, we have

$$
\mathbb{P}\left(\left|\widehat{\beta}_{j, k}-\beta_{j, k}\right| \geq(\kappa / 2) \theta 2^{j} \sqrt{\frac{\rho_{n} \ln \left(n / \rho_{n}\right)}{n}}\right) \leq 2\left(\frac{\rho_{n}}{n}\right)^{2},
$$

where $\theta$ is defined by (4.4).

The technical details can be found in the proof of Proposition 6.2.

The next subsections are devoted to the description of our main estimators for $f_{\nu}$.

\subsection{Wavelet linear estimator}

We define the linear estimator $\widehat{f}_{\nu}^{l}$ by

$$
\widehat{f}_{\nu}^{l}(x)=\sum_{k=0}^{2^{j_{0}}-1} \widehat{\alpha}_{j_{0}, k} \phi_{j_{0}, k}(x), \quad x \in[0,1],
$$

where $\widehat{\alpha}_{j, k}$ is defined by (4.2) and $j_{0}$ is an integer which will be chosen later (see Theorem 5.1).

imsart-generic ver. 2009/12/15 file: bj4_yogen_rev.tex date: December 12, 2013 
In the case $m=1, w_{1}(1)=\ldots=w_{1}(n)=1$ and $f_{\nu}=f_{1}, \hat{f}_{\nu}^{l}$ becomes the linear wavelet estimator developed in Abbaszadeh et al. (2012).

For a survey on linear wavelet estimators for various density models (including the density model under multiplicative censoring), we refer to Chaubey et al. (2011).

\subsection{Wavelet hard thresholding estimator}

We define the hard thresholding estimator $\widehat{f}_{\nu}^{h}$ by

$$
\widehat{f}_{\nu}^{h}(x)=\sum_{k=0}^{2^{\tau}-1} \widehat{\alpha}_{\tau, k} \phi_{\tau, k}(x)+\sum_{j=\tau}^{j_{1}} \sum_{k=0}^{2^{j}-1} \widehat{\beta}_{j, k} \mathbf{1}_{\left\{\left|\widehat{\beta}_{j, k}\right| \geq \kappa \lambda_{j}\right\}} \psi_{j, k}(x), \quad x \in[0,1],
$$

where $\widehat{\alpha}_{j, k}$ is defined by (4.2), $\widehat{\beta}_{j, k}$ by $(4.3), \tau$ is the primary resolution level of the wavelet basis $\mathcal{B}, j_{1}$ is the integer satisfying

$$
\frac{1}{2}\left(\frac{n}{\rho_{n}}\right)^{1 / 3}<2^{j_{1}} \leq\left(\frac{n}{\rho_{n}}\right)^{1 / 3}
$$

$\kappa \geq 2+8 / 3+2 \sqrt{4+16 / 9}$,

$$
\lambda_{j}=\theta 2^{j} \sqrt{\frac{\rho_{n} \ln \left(n / \rho_{n}\right)}{n}} .
$$

and $\theta$ is defined by (4.4).

The definitions of the thresholds $\eta_{j}$ and $\lambda_{j}$ are based on theoretical considerations; they are chosen in such a way that the MISE of $\widehat{f}_{\nu}^{h}$ is as small as possible, while being independent of $f_{\nu}$ (and its degree of smoothness). This independence makes $\widehat{f}_{\nu}^{h}$ adaptive. Technical details can be found in the proof of Theorem 6.1.

The idea of the considered thresholding is to set to zero all wavelet coefficients that are less than a particular threshold and to estimate the others. The resulting estimators often possess optimal (or near-optimal) rates of convergence in the minimax sense. Moreover, they are typically implemented via fast algorithms which makes them appealing in practice. For the constructions of hard thresholding wavelet estimators in the standard density model, see e.g. Donoho et al. (1996), Delyon and Juditsky (1996) and Härdle et al. (1998), and for the standard density mixture model, see Pokhyl'ko (2005). For the multiplicative censoring density model from biased data, see Abbaszadeh et al. (2012). To the best of our knowledge, $\widehat{f}_{\nu}^{h}$ is the first adaptive wavelet estimator proposed for the density estimation from mixtures under multiplicative censoring. 


\section{Results}

Theorem 5.1 below explores the asymptotic performance of $\widehat{f}_{\nu}^{l}$ under the MISE over Besov balls $B_{p, r}^{s}(M)$. We distinguish the "homogeneous zone" corresponding to $p \geq 2$ and the "inhomogeneous zone" corresponding to $p \in[1,2)$, following the classification of Härdle et al. (1998).

Theorem 5.1. Consider (1.1) under the assumptions of Section 2.

(i) Suppose that $f_{\nu} \in B_{p, r}^{s}(M)$ with $s \in(0, N), p \geq 2$ and $r \geq 1$. Let $\widehat{f}_{\nu}^{l}$ be given by (4.5) with $j_{0}$ satisfying

$$
\frac{1}{2}\left(\frac{n}{\rho_{n}}\right)^{1 /(2 s+3)}<2^{j_{0}} \leq\left(\frac{n}{\rho_{n}}\right)^{1 /(2 s+3)} .
$$

Then there exists a constant $C>0$ such that

$$
\mathbb{E}\left(\left\|\widehat{f}_{\nu}-f_{\nu}\right\|_{2}^{2}\right) \leq C\left(\frac{\rho_{n}}{n}\right)^{2 s /(2 s+3)} .
$$

(ii) Suppose that $f_{\nu} \in B_{p, r}^{s}(M)$ with $s \in(1 / p, N), p \in[1,2)$ and $r \geq 1$. Let $\widehat{f}_{\nu}^{l}$ be given by (4.5) with $j_{0}$ satisfying

$$
\frac{1}{2}\left(\frac{n}{\rho_{n}}\right)^{1 /\left(2 s_{*}+3\right)}<2^{j_{0}} \leq\left(\frac{n}{\rho_{n}}\right)^{1 /\left(2 s_{*}+3\right)},
$$

where $s_{*}=s+1 / 2-1 / p$.

Then there exists a constant $C>0$ such that

$$
\mathbb{E}\left(\left\|\widehat{f}_{\nu}-f_{\nu}\right\|_{2}^{2}\right) \leq C\left(\frac{\rho_{n}}{n}\right)^{2 s_{*} /\left(2 s_{*}+3\right)} .
$$

The proof of Theorem 5.1 (see $\S 6$ ) uses moment inequalities on (4.2) and (4.3), and a suitable decomposition of the MISE.

Let us mention that, in each zone of the Besov balls, the integer $j_{0}$ is chosen to minimize the MISE of $\hat{f}_{\nu}^{l}$ in a nonadaptive way.

Remark 5.1. The variability of the performance of linear estimators according to the homogeneous nature of the zones of the Besov balls is a well known phenomenon. In particular, in the context of the standard nonparametric smoothing (such as for density and regression), the rates are often non-optimal (in the minimax sense) over the inhomogeneous zone. We refer to Härdle et al. (1998), p.148.

Theorem 5.2 given next investigates the asymptotic performance of $\widehat{f}_{\nu}^{h}$ under the MISE over Besov balls (the homogeneous and inhomogeneous zones are considered simultaneously). 
Theorem 5.2. Consider (1.1) under the assumptions of Section 2. Let $\hat{f}_{\nu}^{h}$ be given by (4.6). Suppose that $f_{\nu} \in B_{p, r}^{s}(M)$ with $r \geq 1,\{p \geq 2$ and $s \in(0, N)\}$ or $\{p \in[1,2)$ and $s \in(3 / p, N)\}$. Then there exists a constant $C>0$ such that

$$
\mathbb{E}\left(\left\|\widehat{f}_{\nu}^{h}-f_{\nu}\right\|_{2}^{2}\right) \leq C\left(\frac{\rho_{n} \ln \left(n / \rho_{n}\right)}{n}\right)^{2 s /(2 s+3)} .
$$

The proof of Theorem 5.2 uses a general result derived to Chesneau (2011b) (see the proof of his Theorem 2).

Theorem 5.2 shows that, in addition to being adaptive, $\hat{f}_{\nu}^{h}$ attains a sharp rate of convergence. More specifically, adapting the terminology of Härdle et al. (1998),

- for the homogeneous zone $(p \geq 2): \hat{f}_{\nu}^{h}$ attains the same rate of convergence to $\widehat{f}_{\nu}^{l}$, i.e. $v_{n}=\left(\rho_{n} / n\right)^{2 s /(2 s+3)}$, up to the logarithmic term $\left(\ln \left(n / \rho_{n}\right)\right)^{2 s /(2 s+3)}$.

- for the inhomogeneous zone $(p \in[1,2)): \widehat{f}_{\nu}^{h}$ attains a better rate of convergence to $\widehat{f}_{\nu}^{l}$.

Remark 5.2. Let us mention that, for the standard nonparametric models, other types of adaptive procedures are able to remove the extra logarithmic term in the rate of convergence, for example,

- the Lepski procedure [see e.g. Lepski (1990, 1991, 1992) and Lepski and Spokoiny (1995)], and

- the BlockJS procedure [see e.g. Cai (1999, 2002)].

Although the adaptation of these procedures to our statistical problem is possible, their asymptotic properties are not established; new important theoretical difficulties appear and it is not immediately clear how to solve them.

Remark 5.3. Suppose that the smallest eigenvalue of the matrix $\Gamma_{n}$ (given in (2.2)) is larger than or equal to $K$ with $K \in(0,1)$. Then Lemma 6.1 of Autin and Pouet (2011) gives

$$
\frac{1}{n} \sum_{d=1}^{m} \sum_{i=1}^{n} a_{d}^{2}(i) \leq \frac{m}{K} .
$$

Hence $\rho_{n} \leq m / K$ and the rate of convergence in Theorem 5.2 becomes $(\ln n / n)^{2 s /(2 s+3)}$. It corresponds to the optimal one (up to a logarithmic term) for the standard multiplicative density model [see Andersen and Hansen(2001)].

Remark 5.4. Suppose that there exists a constant $C>0$ satisfying

$$
\max _{d \in\{1, \ldots, m\}} \max _{i \in\{1, \ldots, n\}}\left|a_{d}(i)\right| \leq C .
$$

Then, with $j_{1}$ such that

$$
\frac{c_{*}}{2}\left(\frac{n}{\ln n}\right)^{1 / 3} \leq 2^{j_{1}} \leq c_{*}\left(\frac{n}{\ln n}\right)^{1 / 3}
$$

imsart-generic ver. 2009/12/15 file: bj4_yogen_rev.tex date: December 12, 2013 
for a suitable constant $c_{*}$, the indicator function in (4.3) is equal to one and Theorem 5.2 can be proved with the rate of convergence $(\ln n / n)^{2 s /(2 s+3)}$.

Conclusion and perspectives. We construct a new adaptive wavelet estimator to estimate the function $f_{\nu}$ from (1.2). Under mild assumptions, we prove that it attains a sharp rate of convergence for a wide class of functions. Possible perspectives are to

- determine the optimal lower bound of MISE for (1.1), perhaps using the Fano Lemma presented in Tsybakov (2004). However, it is not immediately clear how to extend this to the mixture models.

- improve the estimation of $f_{\nu}$ by removing the extra logarithmic term (see Remark 5.2),

- investigate the case where the distributions of $I_{1}, \ldots, I_{n}$ are unknown,

- consider other estimation problems as the adaptive estimation of the $q$-th derivatives of $f_{\nu}$ (as in Prakasa Rao (1996) for the standard density model and in Prakasa Rao (2010) for the standard mixture model).

All these aspects need further investigations that we leave for a future work.

\section{Proofs}

In this section, we consider (1.1) under the assumptions of Section 2. Moreover, $C$ denotes any constant that does not depend on $j, k$ and $n$. Its value may change from one term to another and may depend on $\phi$ or $\psi$.

\subsection{Proof of Proposition 4.1}

Proof of Proposition 4.1. Recall that the density of $Y_{i}$ can be expressed in terms of $h_{i}$ in the following manner:

$$
g_{i}(x)=\int_{x}^{1} \frac{h_{i}(y)}{y} d y, \quad x \in[0,1] .
$$

So $h_{i}(x)=-x g_{i}^{\prime}(x), x \in[0,1]$ a.s.. It follows from integration by parts, $g_{i}(1)=0$, and $\phi_{j, k}(0)$ and $\phi_{j, k}(1)$ exist, that

$$
\begin{aligned}
\int_{0}^{1} & h_{i}(x) \phi_{j, k}(x) d x=-\int_{0}^{1} x g_{i}^{\prime}(x) \phi_{j, k}(x) d x \\
= & -\left[x g_{i}(x) \phi_{j, k}(x)\right]_{0}^{1}+\int_{0}^{1} g_{i}(x)\left(\phi_{j, k}(x)+x\left(\phi_{j, k}\right)^{\prime}(x)\right) d x \\
\quad= & \int_{0}^{1} g_{i}(x) T\left(\phi_{j, k}\right)(x) d x=\mathbb{E}\left(T\left(\phi_{j, k}\right)\left(Y_{i}\right)\right),
\end{aligned}
$$

where $T$ denotes the operator in (4.1). 
Using (6.1), (1.2) and (2.3), we obtain

$$
\begin{aligned}
\mathbb{E}\left(\widehat{\alpha}_{j, k}\right) & =\frac{1}{n} \sum_{i=1}^{n} a_{\nu}(i) \mathbb{E}\left(T\left(\phi_{j, k}\right)\left(Y_{i}\right)\right) \\
& =\frac{1}{n} \sum_{i=1}^{n} a_{\nu}(i) \int_{0}^{1} h_{i}(x) \phi_{j, k}(x) d x \\
& =\sum_{d=1}^{m} \int_{0}^{1} f_{d}(x) \phi_{j, k}(x) d x\left(\frac{1}{n} \sum_{i=1}^{n} a_{\nu}(i) w_{d}(i)\right) \\
& =\int_{0}^{1} f_{\nu}(x) \phi_{j, k}(x) d x=\alpha_{j, k} .
\end{aligned}
$$

Similarly, taking $\psi$ instead of $\phi$, we prove the second point that completes the proof of Proposition 4.1.

\subsection{Proofs of Theorems 5.1 and 5.2}

\section{Proof of Theorem 5.1.}

(i) We expand the function $f_{\nu}$ on $\mathcal{B}$ at the level $j_{0}$ given in (5.1):

$$
f_{\nu}(x)=\sum_{k=0}^{2^{j_{0}}-1} \alpha_{j_{0}, k} \phi_{j_{0}, k}(x)+\sum_{j=j_{0}}^{\infty} \sum_{k=0}^{2^{j}-1} \beta_{j, k} \psi_{j, k}(x),
$$

where $\alpha_{j_{0}, k}=\int_{0}^{1} f_{\nu}(x) \phi_{j_{0}, k}(x) d x$ and $\beta_{j, k}=\int_{0}^{1} f_{\nu}(x) \psi_{j, k}(x) d x$.

We can write

$$
\mathbb{E}\left(\left\|\widehat{f}_{\nu}^{l}-f_{\nu}\right\|_{2}^{2}\right)=\sum_{k=0}^{2^{j 0}-1} \mathbb{E}\left(\left(\widehat{\alpha}_{j_{0}, k}-\alpha_{j_{0}, k}\right)^{2}\right)+\sum_{j=j_{0}}^{\infty} \sum_{k=0}^{2^{j}-1} \beta_{j, k}^{2} .
$$

Let us now bound the first term. Since, by Proposition 4.1, $\widehat{\alpha}_{j, k}$ is an unbiased estimator of $\alpha_{j, k}$, we have

$$
\begin{aligned}
\mathbb{E}\left(\left(\widehat{\alpha}_{j_{0}, k}-\alpha_{j_{0}, k}\right)^{2}\right) & =\frac{1}{n^{2}} \sum_{i=1}^{n} a_{\nu}^{2}(i) \mathbb{V}\left(T\left(\phi_{j_{0}, k}\right)\left(Y_{i}\right)\right) \\
& \leq \frac{1}{n^{2}} \sum_{i=1}^{n} a_{\nu}^{2}(i) \mathbb{E}\left(\left(T\left(\phi_{j_{0}, k}\right)\left(Y_{i}\right)\right)^{2}\right)
\end{aligned}
$$

By the definition of $T$ in (4.1), for any $i \in\{1, \ldots, n\}$, we have

$$
\mathbb{E}\left(\left(T\left(\phi_{j_{0}, k}\right)\left(Y_{i}\right)\right)^{2}\right) \leq 2(A+B),
$$

where

$$
A=\mathbb{E}\left(\phi_{j_{0}, k}^{2}\left(Y_{i}\right)\right), \quad B=\mathbb{E}\left(Y_{i}^{2}\left(\left(\phi_{j_{0}, k}\right)^{\prime}\left(Y_{i}\right)\right)^{2}\right)
$$


Since, by $(2.1), \max _{i \in\{1, \ldots, n\}} \sup _{x \in[0,1]} g_{i}(x) \leq C_{*}$, we have

$$
A=\int_{0}^{1} \phi_{j_{0}, k}^{2}(x) g_{i}(x) d x \leq C_{*} \int_{0}^{1} \phi_{j_{0}, k}^{2}(x) d x=C_{*} .
$$

On the other hand, using again $(2.1),\left(\phi_{j_{0}, k}\right)^{\prime}(x)=2^{3 j_{0} / 2} \phi^{\prime}\left(2^{j_{0}} x-k\right)$, making the change of variables $y=2^{j_{0}} x-k$ and owing to $\operatorname{supp}(\phi) \subseteq$ $[1-N, N]$, we obtain

$$
\begin{aligned}
B & =\int_{0}^{1} x^{2}\left(\left(\phi_{j_{0}, k}\right)^{\prime}(x)\right)^{2} g_{i}(x) d x \leq C_{*} \int_{0}^{1} x^{2}\left(\left(\phi_{j_{0}, k}\right)^{\prime}(x)\right)^{2} d x \\
& \leq C_{*} \int_{0}^{1}\left(\left(\phi_{j_{0}, k}\right)^{\prime}(x)\right)^{2} d x=C_{*} 2^{3 j} \int_{0}^{1}\left(\phi^{\prime}\left(2^{j_{0}} x-k\right)\right)^{2} d x \\
& \leq C_{*} 2^{2 j_{0}} \int_{1-N}^{N}\left(\phi^{\prime}(y)\right)^{2} d y .
\end{aligned}
$$

Hence

$$
\mathbb{E}\left(\left(T\left(\phi_{j_{0}, k}\right)\left(Y_{i}\right)\right)^{2}\right) \leq C_{o} 2^{2 j_{0}},
$$

where $C_{o}=2 C_{*}\left(1+\int_{1-N}^{N}\left(\phi^{\prime}(y)\right)^{2} d y\right)$.

Therefore

$$
\mathbb{E}\left(\left(\widehat{\alpha}_{j_{0}, k}-\alpha_{j_{0}, k}\right)^{2}\right) \leq C 2^{2 j_{0}} \frac{1}{n}\left(\frac{1}{n} \sum_{i=1}^{n} a_{\nu}^{2}(i)\right)=C 2^{2 j_{0}} \frac{\rho_{n}}{n} .
$$

For $j_{0}$ satisfying (5.1) it holds

$$
\sum_{k=0}^{2^{j_{0}}-1} \mathbb{E}\left(\left(\widehat{\alpha}_{j_{0}, k}-\alpha_{j_{0}, k}\right)^{2}\right) \leq C 2^{j_{0}} 2^{2 j_{0}} \frac{\rho_{n}}{n} \leq C\left(\frac{\rho_{n}}{n}\right)^{2 s /(2 s+3)} .
$$

On the other hand, for $p \geq 2$, we have $f_{\nu} \in B_{p, r}^{s}(M) \subseteq B_{2, \infty}^{s}(M)$ [see Härdle et al. (1998), Corollary 9.2], which implies

$$
\sum_{j=j_{0}}^{\infty} \sum_{k=0}^{2^{j}-1} \beta_{j, k}^{2} \leq C 2^{-2 j_{0} s} \leq C\left(\frac{\rho_{n}}{n}\right)^{2 s /(2 s+3)} .
$$

It follows from (6.2), (6.4) and (6.5) that

$$
\mathbb{E}\left(\left\|\widehat{f}_{\nu}^{l}-f_{\nu}\right\|_{2}^{2}\right) \leq C\left(\frac{\rho_{n}}{n}\right)^{2 s /(2 s+3)} .
$$

The part (i) of Theorem 5.1 is proved.

(ii) Let $p \in[1,2)$. The proof is similar to (i) with $s_{*}$ instead of $s$ : we use the expansion (6.2) at the level $j_{0}$ given in (5.2), and the inclusion $B_{p, r}^{s}(M) \subseteq$ $B_{2, \infty}^{s_{*}}(M)$ (thanks to $p \in[1,2)$ and the definition of $s_{*}$, i.e. $s_{*}=s+1 / 2-$ $1 / p$, [see Härdle et al. (1998), Corollary 9.2]), we obtain the desired result. 
This completes the proof of Theorem 5.1.

Proof of Theorem 5.2. First of all, we present a general result (see Theorem 6.1) derived from Theorem 2 of Chesneau (2011b). Its proof is given in $\S 6.2$. In this theorem, we consider a more general form of the hard thresholding estimator $\hat{f}_{\nu}^{h}$ denoted by $\hat{f}_{H}$ for estimating an unknown function $f \in \mathbb{L}^{2}([0,1])$ from $n$ independent random variables $W_{1}, \ldots, W_{n}$ defined on a probability space $(\Omega, \mathcal{A}, \mathbb{P})$ (We use the notations of Section 3 ):

$$
\widehat{f}_{H}(x)=\sum_{k=0}^{2^{\tau}-1} \widehat{\alpha}_{\tau, k} \phi_{\tau, k}(x)+\sum_{j=\tau}^{j_{1}} \sum_{k=0}^{2^{j}-1} \widehat{\beta}_{j, k} \mathbf{1}_{\left\{\left|\widehat{\beta}_{j, k}\right| \geq \kappa \vartheta_{j}\right\}} \psi_{j, k}(x),
$$

where

$$
\begin{gathered}
\widehat{\alpha}_{j, k}=\frac{1}{v_{n}} \sum_{i=1}^{n} q_{i}\left(\phi_{j, k}, W_{i}\right), \\
\widehat{\beta}_{j, k}=\frac{1}{v_{n}} \sum_{i=1}^{n} q_{i}\left(\psi_{j, k}, W_{i}\right) \mathbf{1}_{\left\{\left|q_{i}\left(\psi_{j, k}, W_{i}\right)\right| \leq \varsigma_{j}\right\}}, \\
\varsigma_{j}=\theta_{\psi} 2^{\omega j} \frac{v_{n}}{\sqrt{\mu_{n} \ln \mu_{n}}} \\
\vartheta_{j}=\theta_{\psi} 2^{\omega j} \sqrt{\frac{\ln \mu_{n}}{\mu_{n}}},
\end{gathered}
$$

$\kappa \geq 2+8 / 3+2 \sqrt{4+16 / 9}$ and $j_{1}$ is the integer satisfying

$$
\frac{1}{2} \mu_{n}^{1 /(2 \omega+1)}<2^{j_{1}} \leq \mu_{n}^{1 /(2 \omega+1)} .
$$

Here, we suppose that there exist

- $n$ functions $q_{1}, \ldots, q_{n}$ with $q_{i}: \mathbb{L}^{2}([0,1]) \times \mathbb{R} \rightarrow \mathbb{R}$ for any $i \in\{1, \ldots, n\}$,

- two sequences of real numbers $\left(v_{n}\right)_{n \in \mathbb{N}}$ and $\left(\mu_{n}\right)_{n \in \mathbb{N}}$ satisfying $\lim _{n \rightarrow \infty} v_{n}=$ $\infty$ and $\lim _{n \rightarrow \infty} \mu_{n}=\infty$

such that, for $\gamma \in\{\phi, \psi\}$,

(A1). any integer $j \geq \tau$ and any $k \in\left\{0, \ldots, 2^{j}-1\right\}$,

$$
\mathbb{E}\left(\frac{1}{v_{n}} \sum_{i=1}^{n} q_{i}\left(\gamma_{j, k}, W_{i}\right)\right)=\int_{0}^{1} f(x) \gamma_{j, k}(x) d x .
$$

(A2). there exist two constants, $\theta_{\gamma}>0$ and $\omega \geq 0$, such that, for any integer $j \geq \tau$ and any $k \in\left\{0, \ldots, 2^{j}-1\right\}$,

$$
\sum_{i=1}^{n} \mathbb{E}\left(\left(q_{i}\left(\gamma_{j, k}, W_{i}\right)\right)^{2}\right) \leq \theta_{\gamma}^{2} 2^{2 \omega j} \frac{v_{n}^{2}}{\mu_{n}}
$$


Remark 6.1. The estimators $\widehat{\alpha}_{j, k}$ and $\widehat{\beta}_{j, k}$ defined in the above theorem coincide with those in (4.2) and (4.3), respectively by choosing $v_{n}=n$. The particular form of the estimator chosen will be clear from the context.

Now we are ready to state the theorem required for proving Theorem 5.2.

Theorem 6.1. Let $\hat{f}_{H}$ be (6.6) under (A1) and (A2). Suppose that $f \in B_{p, r}^{s}(M)$ with $r \geq 1,\{p \geq 2$ and $s \in(0, N)\}$ or $\{p \in[1,2)$ and $s \in((2 \omega+1) / p, N)\}$. Then there exists a constant $C>0$ such that

$$
\mathbb{E}\left(\left\|\widehat{f}_{H}-f\right\|_{2}^{2}\right) \leq C\left(\frac{\ln \mu_{n}}{\mu_{n}}\right)^{2 s /(2 s+2 \omega+1)} .
$$

Remark 6.2. Theorem 6.1 is still valid

- with $j_{1}$ such that

$$
\frac{c_{*}}{2}\left(\frac{\mu_{n}}{\ln \mu_{n}}\right)^{1 /(2 \omega+1)}<2^{j_{1}} \leq c_{*}\left(\frac{\mu_{n}}{\ln \mu_{n}}\right)^{1 /(2 \omega+1)},
$$

$c_{*}>0$, instead of (6.11).

- without $\theta_{\psi}$ in the definitions of (6.9) and (6.10), but with $\kappa \geq 2 \theta_{\psi}^{2}+8 / 3+$ $2 \sqrt{4 \theta_{\psi}^{2}+16 / 9}$.

Let us now investigate the assumptions (A1) and (A2) of Theorem 6.1 for the model (1.1). Set, for any $\gamma \in\{\phi, \psi\}$,

$$
q_{i}\left(\gamma_{j, k}, y\right)=a_{\nu}(i) T\left(\gamma_{j, k}\right)(y) .
$$

By Proposition 4.1, for any $\gamma \in\{\phi, \psi\}$, we have

$$
\mathbb{E}\left(\frac{1}{n} \sum_{i=1}^{n} q_{i}\left(\gamma_{j, k}, Y_{i}\right)\right)=\int_{0}^{1} f_{\nu}(x) \gamma_{j, k}(x) d x .
$$

Proceeding as in (6.3), we obtain

$$
\frac{1}{n^{2}} \sum_{i=1}^{n} \mathbb{E}\left(\left(q_{i}\left(\gamma_{j, k}, Y_{i}\right)\right)^{2}\right) \leq \theta_{\gamma}^{2} 2^{2 j} \frac{1}{n^{2}} \sum_{i=1}^{n} a_{\nu}^{2}(i)=\theta_{\gamma}^{2} 2^{2 j} \frac{\rho_{n}}{n},
$$

with

$$
\theta_{\gamma}=\sqrt{2 C_{*}\left(1+\int_{1-N}^{N}\left(\gamma^{\prime}(y)\right)^{2} d y\right)} .
$$

Thus the assumptions (A1) and (A2) in Theorem 6.1 are satisfied with, for any $i \in\{1, \ldots, n\}$,

$$
W_{i}=Y_{i}=U_{i} X_{i}, \quad q_{i}\left(\gamma_{j, k}, y\right)=a_{\nu}(i) T\left(\gamma_{j, k}\right)(y),
$$

imsart-generic ver. 2009/12/15 file: bj4_yogen_rev.tex date: December 12, 2013 
$v_{n}=n, \omega=1, \mu_{n}=n / \rho_{n}$ and $\theta_{\gamma}$ is (6.12).

This implies the following result:

Let $f_{\nu} \in B_{p, r}^{s}(M)$ with $r \geq 1,\{p \geq 2$ and $s \in(0, N)\}$ or $\{p \in[1,2)$ and $s \in(3 / p, N)\}$, then the hard thresholding estimator $\widehat{f}_{\nu}^{h}(4.6)$ satisfies

$$
\mathbb{E}\left(\left\|\widehat{f}_{\nu}^{h}-f_{\nu}\right\|_{2}^{2}\right) \leq C\left(\frac{\rho_{n} \ln \left(n / \rho_{n}\right)}{n}\right)^{2 s /(2 s+3)} .
$$

This completes the proof of Theorem 5.2.

\subsection{Proof of Theorem 6.1}

The proof of the theorem is broken into two major parts. In the first part, we present two auxiliary results on the statistical properties of (6.7) and (6.8). Then, following the idea of Härdle et al. (1998), Proof of Proposition 10.3, these results are used in the second part on a suitable decomposition of the MISE of $\widehat{f}_{H}$.

\section{Part I: Auxiliary results}

Proposition 6.1. Consider $\widehat{\beta}_{j, k}$ as defined in (6.8). We have

$$
\left|\widehat{\beta}_{j, k}-\beta_{j, k}\right| \leq \frac{1}{v_{n}}\left|\sum_{i=1}^{n} U_{i}\right|+\vartheta_{j},
$$

where

$$
U_{i}=q_{i}\left(\psi_{j, k}, W_{i}\right) \mathbf{1}_{\left\{\left|q_{i}\left(\psi_{j, k}, W_{i}\right)\right| \leq \varsigma_{j}\right\}}-\mathbb{E}\left(q_{i}\left(\psi_{j, k}, W_{i}\right) \mathbf{1}_{\left\{\left|q_{i}\left(\psi_{j, k}, W_{i}\right)\right| \leq \varsigma_{j}\right\}}\right) .
$$

Proof of Proposition 6.1. By (A1), we have

$$
\begin{aligned}
\beta_{j, k} & =\mathbb{E}\left(\frac{1}{v_{n}} \sum_{i=1}^{n} q_{i}\left(\psi_{j, k}, W_{i}\right)\right) \\
& =\frac{1}{v_{n}} \sum_{i=1}^{n} \mathbb{E}\left(q_{i}\left(\psi_{j, k}, W_{i}\right) \mathbf{1}_{\left\{\left|q_{i}\left(\psi_{j, k}, W_{i}\right)\right| \leq \varsigma_{j}\right\}}\right) \\
& +\frac{1}{v_{n}} \sum_{i=1}^{n} \mathbb{E}\left(q_{i}\left(\psi_{j, k}, W_{i}\right) \mathbf{1}_{\left\{\left|q_{i}\left(\psi_{j, k}, W_{i}\right)\right|>\varsigma_{j}\right\}}\right) .
\end{aligned}
$$

Hence

$$
\begin{aligned}
& \left|\widehat{\beta}_{j, k}-\beta_{j, k}\right| \\
& \quad \leq \frac{1}{v_{n}}\left|\sum_{i=1}^{n} U_{i}\right|+\frac{1}{v_{n}} \sum_{i=1}^{n} \mathbb{E}\left(\left|q_{i}\left(\psi_{j, k}, W_{i}\right)\right| \mathbf{1}_{\left\{\left|q_{i}\left(\psi_{j, k}, W_{i}\right)\right|>\varsigma_{j}\right\}}\right),
\end{aligned}
$$

imsart-generic ver. 2009/12/15 file: bj4_yogen_rev.tex date: December 12, 2013 
where $U_{1}, \ldots, U_{n}$ are given by (6.13).

The Markov inequality and (A2) imply that

$$
\begin{aligned}
& \frac{1}{v_{n}} \sum_{i=1}^{n} \mathbb{E}\left(\left|q_{i}\left(\psi_{j, k}, W_{i}\right)\right| \mathbf{1}_{\left\{\left|q_{i}\left(\psi_{j, k}, W_{i}\right)\right|>\varsigma_{j}\right\}}\right) \\
& \quad \leq \frac{1}{\varsigma_{j} v_{n}} \sum_{i=1}^{n} \mathbb{E}\left(\left(q_{i}\left(\psi_{j, k}, W_{i}\right)\right)^{2}\right) \leq \frac{1}{\varsigma_{j}} \theta_{\psi}^{2} 2^{2 \omega j} \frac{v_{n}}{\mu_{n}}
\end{aligned}
$$

Using the definitions of $\varsigma_{j}$ and $\vartheta_{j}$ from (6.9) and (6.10) respectively, the right hand side of the above inequality becomes

$$
\frac{1}{\theta_{\psi} 2^{\omega j} \frac{v_{n}}{\sqrt{\mu_{n} \ln \mu_{n}}}} \theta_{\psi}^{2} 2^{2 \omega j} \frac{v_{n}}{\mu_{n}}=\theta_{\psi} 2^{\omega j} \sqrt{\frac{\ln \mu_{n}}{\mu_{n}}}=\vartheta_{j}
$$

that completes the proof of Proposition 6.1.

Proposition 6.2. Consider $\widehat{\alpha}_{j, k}$ and $\widehat{\beta}_{j, k}$ as defined in (6.7) and (6.8), respectively.

(i) There exists a constant $C>0$ such that

$$
\mathbb{E}\left(\left(\widehat{\alpha}_{j, k}-\alpha_{j, k}\right)^{2}\right) \leq C 2^{2 \omega j} \frac{1}{\mu_{n}} .
$$

(ii) There exists a constant $C>0$ such that

$$
\mathbb{E}\left(\left(\widehat{\beta}_{j, k}-\beta_{j, k}\right)^{4}\right) \leq C 2^{4 \omega j}\left(\frac{\ln \mu_{n}}{\mu_{n}}\right)^{2} .
$$

(iii) For any $\kappa \geq 2+8 / 3+2 \sqrt{4+16 / 9}$, we have

$$
\mathbb{P}\left(\left|\widehat{\beta}_{j, k}-\beta_{j, k}\right| \geq \kappa \vartheta_{j} / 2\right) \leq 2 \frac{1}{\mu_{n}^{2}} .
$$

\section{Proof of Proposition 6.2.}

(i) Noting that $\widehat{\alpha}_{j, k}$ is an unbiased estimator of $\alpha_{j, k}$, by (A1), the independence of $W_{1}, \ldots, W_{n}$ and (A2) yield

$$
\begin{aligned}
\mathbb{E}\left(\left(\widehat{\alpha}_{j, k}-\alpha_{j, k}\right)^{2}\right) & =\mathbb{V}\left(\widehat{\alpha}_{j, k}\right)=\frac{1}{v_{n}^{2}} \sum_{i=1}^{n} \mathbb{V}\left(q_{i}\left(\phi_{j, k}, W_{i}\right)\right) \\
& \leq \frac{1}{v_{n}^{2}} \sum_{i=1}^{n} \mathbb{E}\left(\left(q_{i}\left(\phi_{j, k}, W_{i}\right)\right)^{2}\right) \\
& \leq \theta_{\phi}^{2} 2^{2 \omega j} \frac{1}{\mu_{n}} .
\end{aligned}
$$

imsart-generic ver. 2009/12/15 file: bj4_yogen_rev.tex date: December 12, 2013 
(ii) Using Proposition 6.1 we have

$$
\mathbb{E}\left(\left(\widehat{\beta}_{j, k}-\beta_{j, k}\right)^{4}\right) \leq C\left(\frac{1}{v_{n}^{4}} \mathbb{E}\left(\left(\sum_{i=1}^{n} U_{i}\right)^{4}\right)+\vartheta_{j}^{4}\right) .
$$

where $U_{1}, \ldots, U_{n}$ are given by (6.13).

Note that $\mathbb{E}\left(U_{i}\right)=0$ and

$$
\mathbb{E}\left(\left(U_{i}\right)^{4}\right) \leq 16 \varsigma_{j}^{2} \mathbb{E}\left(\left(q_{i}\left(\psi_{j, k}, W_{i}\right)\right)^{2}\right) .
$$

Hence using the Rosenthal inequality (see the Appendix), (A2) and the definition of $\varsigma_{j}$ from (6.9), we have

$$
\begin{aligned}
& \mathbb{E}\left(\left(\sum_{i=1}^{n} U_{i}\right)^{4}\right) \leq C\left(\sum_{i=1}^{n} \mathbb{E}\left(U_{i}^{4}\right)+\left(\sum_{i=1}^{n} \mathbb{E}\left(U_{i}^{2}\right)\right)^{2}\right) \\
& \leq C\left(\varsigma_{j}^{2} \sum_{i=1}^{n} \mathbb{E}\left(\left(q_{i}\left(\psi_{j, k}, W_{i}\right)\right)^{2}\right)+\left(\sum_{i=1}^{n} \mathbb{E}\left(\left(q_{i}\left(\psi_{j, k}, W_{i}\right)\right)^{2}\right)\right)^{2}\right) \\
& \leq C\left(\varsigma_{j}^{2} \theta_{\psi}^{2} 2^{2 \omega j} \frac{v_{n}^{2}}{\mu_{n}}+\theta_{\psi}^{4} 2^{4 \omega j} \frac{v_{n}^{4}}{\mu_{n}^{2}}\right) \\
& \leq C\left(2^{4 \omega j} \frac{v_{n}^{4}}{\mu_{n}^{2} \ln \mu_{n}}+2^{4 \omega j} \frac{v_{n}^{4}}{\mu_{n}^{2}}\right) \leq C 2^{4 \omega j} \frac{v_{n}^{4}}{\mu_{n}^{2}} .
\end{aligned}
$$

It follows from (6.15), (6.16) and the definition of $\vartheta_{j}$ from (6.10) that

$$
\mathbb{E}\left(\left(\widehat{\beta}_{j, k}-\beta_{j, k}\right)^{4}\right) \leq C 2^{4 \omega j}\left(\frac{\ln \mu_{n}}{\mu_{n}}\right)^{2} .
$$

(iii) Proposition 6.1 yields

$$
\mathbb{P}\left(\left|\widehat{\beta}_{j, k}-\beta_{j, k}\right| \geq \kappa \vartheta_{j} / 2\right) \leq \mathbb{P}\left(\left|\sum_{i=1}^{n} U_{i}\right| \geq \kappa_{*} v_{n} \vartheta_{j}\right),
$$

where $U_{1}, \ldots, U_{n}$ are given by $(6.13)$ and $\kappa_{*}=\kappa / 2-1$. Observe that $\mathbb{E}\left(U_{1}\right)=0,\left|U_{1}\right| \leq 2 \varsigma_{j}$ and, by (A2),

$$
\sum_{i=1}^{n} \mathbb{E}\left(U_{i}^{2}\right) \leq \sum_{i=1}^{n} \mathbb{E}\left(\left(q_{i}\left(\psi_{j, k}, W_{i}\right)\right)^{2}\right) \leq \theta_{\psi}^{2} 2^{2 \omega j} \frac{v_{n}^{2}}{\mu_{n}}
$$

Hence, using the Bernstein inequality (see the Appendix) and the defini- 
tions of $\vartheta_{j}$ and $\varsigma_{j}$ from (6.9) (6.10), respectively, we have

$$
\begin{aligned}
& \mathbb{P}\left(\left|\sum_{i=1}^{n} U_{i}\right| \geq \kappa_{*} v_{n} \vartheta_{j}\right) \\
& \leq 2 \exp \left(-\frac{\kappa_{*}^{2} v_{n}^{2} \vartheta_{j}^{2}}{2\left(\sum_{i=1}^{n} \mathbb{E}\left(U_{i}^{2}\right)+\frac{2}{3} \kappa_{*} v_{n} \vartheta_{j} \varsigma_{j}\right)}\right) \\
& \leq 2 \exp \left(-\frac{\kappa_{*}^{2} v_{n}^{2} \theta_{\psi}^{2} 2^{2 \omega j} \frac{\ln \mu_{n}}{\mu_{n}}}{2\left(\theta_{\psi}^{2} 2^{2 \omega j} \frac{v_{n}^{2}}{\mu_{n}}+\frac{2}{3} \kappa_{*} \theta_{\psi}^{2} 2^{2 \omega j} \frac{v_{n}^{2}}{\mu_{n}}\right)}\right) \\
& =2 \mu_{n}^{-\frac{\kappa_{*}^{2}}{2\left(1+\frac{2}{3} \kappa_{*}\right)}} \text {. }
\end{aligned}
$$

Taking $\kappa$ such that $\kappa_{*}^{2} /\left(2\left(1+(2 / 3) \kappa_{*}\right)\right) \geq 2$, i.e. $\kappa \geq 2+8 / 3+2 \sqrt{4+16 / 9}$, we obtain

$$
\mathbb{P}\left(\left|\widehat{\beta}_{j, k}-\beta_{j, k}\right| \geq \kappa \vartheta_{j} / 2\right) \leq 2 \frac{1}{\mu_{n}^{2}}
$$

This completes the proof of Proposition 6.2.

\section{Part II: Proof of the theorem}

We decompose $f$ on the wavelet basis $\mathcal{B}$ as

$$
f(x)=\sum_{k=0}^{2^{\tau}-1} \alpha_{\tau, k} \phi_{\tau, k}(x)+\sum_{j=\tau}^{\infty} \sum_{k=0}^{2^{j}-1} \beta_{j, k} \psi_{j, k}(x),
$$

where $\alpha_{\tau, k}=\int_{0}^{1} f(x) \phi_{\tau, k}(x) d x$ and $\beta_{j, k}=\int_{0}^{1} f(x) \psi_{j, k}(x) d x$. Using the orthonormality of the wavelet basis $\mathcal{B}$, we can decompose the MISE of $\widehat{f}_{H}$ in three terms as:

$$
\mathbb{E}\left(\left\|\widehat{f}_{H}-f\right\|_{2}^{2}\right)=E+F+G
$$

where

$$
E=\sum_{k=0}^{2^{\tau}-1} \mathbb{E}\left(\left(\widehat{\alpha}_{\tau, k}-\alpha_{\tau, k}\right)^{2}\right), \quad F=\sum_{j=\tau}^{j_{1}} \sum_{k=0}^{2^{j}-1} \mathbb{E}\left(\left(\widehat{\beta}_{j, k} \mathbf{1}_{\left\{\left|\widehat{\beta}_{j, k}\right| \geq \kappa \vartheta_{j}\right\}}-\beta_{j, k}\right)^{2}\right)
$$

and

$$
G=\sum_{j=j_{1}+1}^{\infty} \sum_{k=0}^{2^{j}-1} \beta_{j, k}^{2}
$$

We now exhibit the bounds of each of these terms.

Bound for $E$ : 
Proposition 6.2 gives

$$
E \leq C \frac{1}{\mu_{n}} \leq C\left(\frac{\ln \mu_{n}}{\mu_{n}}\right)^{2 s /(2 s+2 \omega+1)} .
$$

Bound for $F$ :

For the sake of simplicity, set $\hat{B}_{j, k}=\widehat{\beta}_{j, k}-\beta_{j, k}$. The term $F$ can be decomposed as

$$
F=F_{1}+F_{2}+F_{3}+F_{4},
$$

where

$$
\begin{gathered}
F_{1}=\sum_{j=\tau}^{j_{1}} \sum_{k=0}^{2^{j}-1} \mathbb{E}\left(\hat{B}_{j, k}^{2} \mathbf{1}_{\left\{\left|\widehat{\beta}_{j, k}\right| \geq \kappa \vartheta_{j},\left|\beta_{j, k}\right|<\kappa \vartheta_{j} / 2\right\}}\right), \\
F_{2}=\sum_{j=\tau}^{j_{1}} \sum_{k=0}^{2^{j}-1} \mathbb{E}\left(\hat{B}_{j, k}^{2} \mathbf{1}_{\left\{\left|\widehat{\beta}_{j, k}\right| \geq \kappa \vartheta_{j},\left|\beta_{j, k}\right| \geq \kappa \vartheta_{j} / 2\right\}}\right), \\
F_{3}=\sum_{j=\tau}^{j_{1}} \sum_{k=0}^{2^{j}-1} \mathbb{E}\left(\beta_{j, k}^{2} \mathbf{1}_{\left\{\left|\widehat{\beta}_{j, k}\right|<\kappa \vartheta_{j},\left|\beta_{j, k}\right| \geq 2 \kappa \vartheta_{j}\right\}}\right)
\end{gathered}
$$

and

$$
F_{4}=\sum_{j=\tau}^{j_{1}} \sum_{k=0}^{2^{j}-1} \mathbb{E}\left(\beta_{j, k}^{2} \mathbf{1}_{\left\{\left|\widehat{\beta}_{j, k}\right|<\kappa \vartheta_{j},\left|\beta_{j, k}\right|<2 \kappa \vartheta_{j}\right\}}\right) .
$$

Bound for $F_{1}+F_{3}$ :

Observe that

$$
\begin{aligned}
& \mathbf{1}_{\left\{\left|\widehat{\beta}_{j, k}\right|<\kappa \vartheta_{j},\left|\beta_{j, k}\right| \geq 2 \kappa \vartheta_{j}\right\}} \leq \mathbf{1}_{\left\{\left|\hat{B}_{j, k}\right|>\kappa \vartheta_{j} / 2\right\}}, \\
& \mathbf{1}_{\left\{\left|\widehat{\beta}_{j, k}\right| \geq \kappa \vartheta_{j},\left|\beta_{j, k}\right|<\kappa \vartheta_{j} / 2\right\}} \leq \mathbf{1}_{\left\{\left|\hat{B}_{j, k}\right|>\kappa \vartheta_{j} / 2\right\}}
\end{aligned}
$$

and

$$
\mathbf{1}_{\left\{\left|\widehat{\beta}_{j, k}\right|<\kappa \vartheta_{j},\left|\beta_{j, k}\right| \geq 2 \kappa \vartheta_{j}\right\}} \leq \mathbf{1}_{\left\{\left|\beta_{j, k}\right| \leq 2\left|\hat{B}_{j, k}\right|\right\}} \cdot
$$

Owing to the previous inequalities, the Cauchy-Schwarz inequality and Proposition 6.2 , we obtain

$$
\begin{aligned}
& F_{1}+F_{3} \leq C \sum_{j=\tau}^{j_{1}} \sum_{k=0}^{2^{j}-1} \mathbb{E}\left(\hat{B}_{j, k}^{2} \mathbf{1}_{\left\{\left|\hat{B}_{j, k}\right|>\kappa \vartheta_{j} / 2\right\}}\right) \\
& \leq C \sum_{j=\tau}^{j_{1}} \sum_{k=0}^{2^{j}-1}\left(\mathbb{E}\left(\hat{B}_{j, k}^{4}\right)\right)^{1 / 2}\left(\mathbb{P}\left(\left|\hat{B}_{j, k}\right|>\kappa \vartheta_{j} / 2\right)\right)^{1 / 2} \\
& \leq C \frac{\ln \mu_{n}}{\mu_{n}^{2}} \sum_{j=\tau}^{j_{1}} 2^{j(1+2 \omega)} \leq C \frac{\ln \mu_{n}}{\mu_{n}} \leq C\left(\frac{\ln \mu_{n}}{\mu_{n}}\right)^{2 s /(2 s+2 \omega+1)} .
\end{aligned}
$$

imsart-generic ver. 2009/12/15 file: bj4_yogen_rev.tex date: December 12, 2013 
Bound for $F_{2}$ :

Using the Cauchy-Schwarz inequality and Proposition 6.2, we obtain

$$
\begin{aligned}
F_{2} & \leq \sum_{j=\tau}^{j_{1}} \sum_{k=0}^{2^{j}-1}\left(\mathbb{E}\left(\hat{B}_{j, k}^{4}\right)\right)^{1 / 2} \mathbf{1}_{\left\{\left|\beta_{j, k}\right| \geq \kappa \vartheta_{j} / 2\right\}} \\
& \leq C \frac{\ln \mu_{n}}{\mu_{n}} \sum_{j=\tau}^{j_{1}} 2^{2 \omega j} \sum_{k=0}^{2^{j}-1} \mathbf{1}_{\left\{\left|\beta_{j, k}\right|>\kappa \vartheta_{j} / 2\right\}} .
\end{aligned}
$$

Let $j_{*}$ be the integer defined by

$$
\frac{1}{2}\left(\frac{\mu_{n}}{\ln \mu_{n}}\right)^{1 /(2 s+2 \omega+1)}<2^{j_{*}} \leq\left(\frac{\mu_{n}}{\ln \mu_{n}}\right)^{1 /(2 s+2 \omega+1)} .
$$

Then $F_{2}$ can be bounded as

$$
F_{2} \leq F_{2,1}+F_{2,2},
$$

where

$$
F_{2,1}=C \frac{\ln \mu_{n}}{\mu_{n}} \sum_{j=\tau}^{j_{*}} 2^{2 \omega j} \sum_{k=0}^{2^{j}-1} \mathbf{1}_{\left\{\left|\beta_{j, k}\right|>\kappa \vartheta_{j} / 2\right\}}
$$

and

$$
F_{2,2}=C \frac{\ln \mu_{n}}{\mu_{n}} \sum_{j=j_{*}+1}^{j_{1}} 2^{2 \omega j} \sum_{k=0}^{2^{j}-1} \mathbf{1}_{\left\{\left|\beta_{j, k}\right|>\kappa \vartheta_{j} / 2\right\}} .
$$

We have

$$
F_{2,1} \leq C \frac{\ln \mu_{n}}{\mu_{n}} \sum_{j=\tau}^{j_{*}} 2^{j(1+2 \omega)} \leq C\left(\frac{\ln \mu_{n}}{\mu_{n}}\right)^{2 s /(2 s+2 \omega+1)} .
$$

For $r \geq 1$ and $p \geq 2$, the Markov inequality, definition of $\vartheta_{j}$ (from (6.10)) and $f \in B_{p, r}^{s}(M) \subseteq B_{2, \infty}^{s}(M)$ (see Härdle et al. (1998), Corollary 9.2) yield

$$
\begin{aligned}
F_{2,2} & \leq C \frac{\ln \mu_{n}}{\mu_{n}} \sum_{j=j_{*}+1}^{j_{1}} 2^{2 \omega j} \frac{1}{\vartheta_{j}^{2}} \sum_{k=0}^{2^{j}-1} \beta_{j, k}^{2} \leq C \sum_{j=j_{*}+1}^{\infty} \sum_{k=0}^{2^{j}-1} \beta_{j, k}^{2} \\
& \leq C \sum_{j=j_{*}+1}^{\infty} 2^{-2 j s} \leq C\left(\frac{\ln \mu_{n}}{\mu_{n}}\right)^{2 s /(2 s+2 \omega+1)} .
\end{aligned}
$$

For $r \geq 1, p \in[1,2)$ and $s>(2 \omega+1) / p$, the Markov inequality, the definition of $\vartheta_{j}, f \in B_{p, r}^{s}(M)$ and $(2 s+2 \omega+1)(2-p) / 2+(s+1 / 2-1 / p+\omega-2 \omega / p) p=2 s$ 
imply that

$$
\begin{aligned}
F_{2,2} & \leq C \frac{\ln \mu_{n}}{\mu_{n}} \sum_{j=j_{*}+1}^{j_{1}} 2^{2 \omega j} \frac{1}{\vartheta_{j}^{p}} \sum_{k=0}^{2^{j}-1}\left|\beta_{j, k}\right|^{p} \\
& \leq C\left(\frac{\ln \mu_{n}}{\mu_{n}}\right)^{(2-p) / 2} \sum_{j=j_{*}+1}^{\infty} 2^{j \omega(2-p)} 2^{-j(s+1 / 2-1 / p) p} \\
& \leq C\left(\frac{\ln \mu_{n}}{\mu_{n}}\right)^{(2-p) / 2} 2^{-j_{*}(s+1 / 2-1 / p+\omega-2 \omega / p) p} \leq C\left(\frac{\ln \mu_{n}}{\mu_{n}}\right)^{2 s /(2 s+2 \omega+1)} .
\end{aligned}
$$

So, for $r \geq 1,\{p \geq 2$ and $s>0\}$ or $\{p \in[1,2)$ and $s>(2 \omega+1) / p\}$, we have

$$
F_{2} \leq C\left(\frac{\ln \mu_{n}}{\mu_{n}}\right)^{2 s /(2 s+2 \omega+1)} .
$$

Bound for $F_{4}$ :

We have

$$
F_{4} \leq \sum_{j=\tau}^{j_{1}} \sum_{k=0}^{2^{j}-1} \beta_{j, k}^{2} \mathbf{1}_{\left\{\left|\beta_{j, k}\right|<2 \kappa \vartheta_{j}\right\}} .
$$

Let $j_{*}$ be the integer (6.22). Then $F_{4}$ can be bound as

$$
F_{4} \leq F_{4,1}+F_{4,2}
$$

where

$$
F_{4,1}=\sum_{j=\tau}^{j_{*}} \sum_{k=0}^{2^{j}-1} \beta_{j, k}^{2} \mathbf{1}_{\left\{\left|\beta_{j, k}\right|<2 \kappa \vartheta_{j}\right\}}, \quad F_{4,2}=\sum_{j=j_{*}+1}^{j_{1}} \sum_{k=0}^{2^{j}-1} \beta_{j, k}^{2} \mathbf{1}_{\left\{\left|\beta_{j, k}\right|<2 \kappa \vartheta_{j}\right\}} .
$$

Observe that, using the definition of $\vartheta_{j}$, we have

$$
F_{4,1} \leq C \sum_{j=\tau}^{j_{*}} 2^{j} \vartheta_{j}^{2}=C \frac{\ln \mu_{n}}{\mu_{n}} \sum_{j=\tau}^{j_{*}} 2^{j(1+2 \omega)} \leq C\left(\frac{\ln \mu_{n}}{\mu_{n}}\right)^{2 s /(2 s+2 \omega+1)} .
$$

Further, for $r \geq 1$ and $p \geq 2$, since $f \in B_{p, r}^{s}(M) \subseteq B_{2, \infty}^{s}(M)$ (see Härdle et al. (1998), Corollary 9.2), we have

$$
F_{4,2} \leq \sum_{j=j_{*}+1}^{\infty} \sum_{k=0}^{2^{j}-1} \beta_{j, k}^{2} \leq C \sum_{j=j_{*}+1}^{\infty} 2^{-2 j s} \leq C\left(\frac{\ln \mu_{n}}{\mu_{n}}\right)^{2 s /(2 s+2 \omega+1)} .
$$

For $r \geq 1, p \in[1,2)$ and $s>(2 \omega+1) / p$, owing to the Markov inequality, the definition of $\vartheta_{j}$ (from (6.10)), $f \in B_{p, r}^{s}(M)$ and $(2 s+2 \omega+1)(2-p) / 2+(s+$ 
$1 / 2-1 / p+\omega-2 \omega / p) p=2 s$, we obtain

$$
\begin{aligned}
F_{4,2} & \leq C \sum_{j=j_{*}+1}^{j_{1}} \vartheta_{j}^{2-p} \sum_{k=0}^{2^{j}-1}\left|\beta_{j, k}\right|^{p} \\
& =C\left(\frac{\ln \mu_{n}}{\mu_{n}}\right)^{(2-p) / 2} \sum_{j=j_{*}+1}^{j_{1}} 2^{j \omega(2-p)} \sum_{k=0}^{2^{j}-1}\left|\beta_{j, k}\right|^{p} \\
& \leq C\left(\frac{\ln \mu_{n}}{\mu_{n}}\right)^{(2-p) / 2} \sum_{j=j_{*}+1}^{\infty} 2^{j \omega(2-p)} 2^{-j(s+1 / 2-1 / p) p} \\
& \leq C\left(\frac{\ln \mu_{n}}{\mu_{n}}\right)^{(2-p) / 2} 2^{-j_{*}(s+1 / 2-1 / p+\omega-2 \omega / p) p} \leq C\left(\frac{\ln \mu_{n}}{\mu_{n}}\right)^{2 s /(2 s+2 \omega+1)} .
\end{aligned}
$$

Thus, for $r \geq 1,\{p \geq 2$ and $s>0\}$ or $\{p \in[1,2)$ and $s>(2 \omega+1) / p\}$, we have

$$
F_{4} \leq C\left(\frac{\ln \mu_{n}}{\mu_{n}}\right)^{2 s /(2 s+2 \omega+1)} .
$$

Therefore it follows from (6.20), (6.21), (6.23) and (6.24) that

$$
F \leq C\left(\frac{\ln \mu_{n}}{\mu_{n}}\right)^{2 s /(2 s+2 \omega+1)} .
$$

Bound for $G$ :

For $r \geq 1$ and $p \geq 2$, we have $f \in B_{p, r}^{s}(M) \subseteq B_{2, \infty}^{s}(M)$. Since $2 s /(2 s+2 \omega+$ 1) $<2 s /(2 \omega+1)$, we have

$$
G \leq C \sum_{j=j_{1}+1}^{\infty} 2^{-2 j s} \leq C\left(\frac{\ln \mu_{n}}{\mu_{n}}\right)^{2 s /(2 \omega+1)} \leq C\left(\frac{\ln \mu_{n}}{\mu_{n}}\right)^{2 s /(2 s+2 \omega+1)} .
$$

For $r \geq 1$ and $p \in[1,2)$, we have $f \in B_{p, r}^{s}(M) \subseteq B_{2, \infty}^{s+1 / 2-1 / p}(M)$ (see Härdle $e t$ al. (1998), Corollary 9.2). Since $s>(2 \omega+1) / p$, we have $(s+1 / 2-1 / p) /(2 \omega+1)>$ $s /(2 s+2 \omega+1)$. Hence

$$
G \leq C \sum_{j=j_{1}+1}^{\infty} 2^{-2 j(s+1 / 2-1 / p)} \leq C\left(\frac{\ln \mu_{n}}{\mu_{n}}\right)^{2(s+1 / 2-1 / p) /(2 \omega+1)} \leq C\left(\frac{\ln \mu_{n}}{\mu_{n}}\right)^{2 s /(2 s+2 \omega+1)} .
$$

Hence, for $r \geq 1,\{p \geq 2$ and $s>0\}$ or $\{p \in[1,2)$ and $s>(2 \omega+1) / p\}$, we have

$$
G \leq C\left(\frac{\ln \mu_{n}}{\mu_{n}}\right)^{2 s /(2 s+2 \omega+1)} .
$$

Combining (6.18), (6.19), (6.25) and (6.26), we have, for any $r \geq 1,\{p \geq 2$ and $s \in(0, N)\}$ or $\{p \in[1,2)$ and $s \in((2 \omega+1) / p, N)\}$,

$$
\mathbb{E}\left(\left\|\widehat{f}_{H}-f\right\|_{2}^{2}\right) \leq C\left(\frac{\ln \mu_{n}}{\mu_{n}}\right)^{2 s /(2 s+2 \omega+1)} .
$$

This completes the proof of Theorem 6.1. 


\section{Appendix}

Lemma 6.1 (Rosenthal's inequality). Let $p \geq 2, n \in \mathbb{N}^{*}$ and $\left(A_{i}\right)_{i \in\{1, \ldots, n\}}$ be $n$ zero mean independent random variables such that, for any $i \in\{1, \ldots, n\}$, $\mathbb{E}\left(\left|A_{i}\right|^{p}\right)<\infty$. Then there exists a constant $C>0$ such that

$$
\mathbb{E}\left(\left|\sum_{i=1}^{n} A_{i}\right|^{p}\right) \leq C\left(\sum_{v=1}^{n} \mathbb{E}\left(\left|A_{i}\right|^{p}\right)+\left(\sum_{i=1}^{n} \mathbb{E}\left(A_{i}^{2}\right)\right)^{p / 2}\right) .
$$

See Rosenthal (1970) for details.

Lemma 6.2 (Bernstein's inequality). Let $n \in \mathbb{N}^{*}$ and $\left(A_{i}\right)_{i \in\{1, \ldots, n\}}$ be $n$ zero mean independent random variables such that there exists a constant $M>0$ satisfying, for any $i \in\{1, \ldots, n\},\left|A_{i}\right| \leq M<\infty$. Then, for any $y>0$, we have

$$
\mathbb{P}\left(\left|\sum_{i=1}^{n} A_{i}\right| \geq y\right) \leq 2 \exp \left(-\frac{y^{2}}{2\left(\sum_{i=1}^{n} \mathbb{E}\left(A_{i}^{2}\right)+\frac{y M}{3}\right)}\right) .
$$

See Petrov (1995) for details.

\section{Acknowledgements}

The authors are thankful to the reviewers for their comments which have helped in improving the presentation.

\section{References}

Abbaszadeh, M., Chesneau, C. and Doosti, H. (2012). Nonparametric estimation of a density under bias and multiplicative censoring via wavelet methods. Statistics and Probability Letters 82 932-941.

Asgharian, M., Carone, M. and Fakoor, V. (2012). Large-sample study of the kernel density estimators under multiplicative censoring. Annals of Statistics 40 159-187.

Akyildiz, I.F., Su, W., Sankarasubramaniam, Y. and Cayirci, E. (2002). Wireless sensor networks: a survey. Computer Networks 38 393-422.

Andersen, K. And Hansen, M. (2001). Multiplicative censoring: density estimation by a series expansion approach. Journal of Statistical Planning and Inference 98 137-155.

Antoniadis, A. (1997). Wavelets in statistics: a review (with discussion). Journal of the Italian Statistical Society 6 97-144.

Autin, F. And Pouet, C. (2011). Test on the components of mixture densities. Stat. \& Risk Modeling 28 389-410.

CAI, T. (1999). Adaptive wavelet estimation: a block thresholding and oracle inequality approach. The Annals of Statistics 27 898-924. 
CAI, T. (2002). On block thresholding in wavelet regression: adaptivity, block size and threshold level. Statistica Sinica 12 1241-1273.

Chabert, M., Ruiz, D. And Tourneret, J.-Y. (2004). Optimal wavelet for abrupt change detection in multiplicative noise. In Proceedings of IEEE International Conference on Acoustics, Speech, and Signal Processing (ICASSP 2004) 2 1089-1092.

Chaubey, Y.P., Chesneau, C. And Doosti, H. (2011). Wavelet Linear Density Estimation: A review. Journal of the Indian Society of Agricultural Statistics 65 169-179.

Chesneau, C. (2011a). Wavelet density estimators for the deconvolution of a component from a mixture, Sankhyā 73-A 245-266.

Chesneau, C. (2011b). Adaptive wavelet estimator for a function and its derivatives in an indirect convolution model, Journal of Statistical Theory and Practice 5 303-326.

Chesneau, C. And Fadili, J. (2012). Adaptive wavelet estimation of a function in an indirect regression model. Advances in Statistical Analysis, 96, 1, $25-46$.

Cohen, A., Daubechies, I., Jawerth, B. and Vial, P. (1993). Wavelets on the interval and fast wavelet transforms. Applied and Computational Harmonic Analysis 24 54-81.

Delyon, B. And Juditsky, A. (1996). On minimax wavelet estimators, Applied Computational Harmonic Analysis. 3 215-228.

Donoho, D. L. And Johnstone, I. M. (1994). Ideal spatial adaptation by wavelet shrinkage. Biometrika 81 425-455.

Donoho, D. L. And Johnstone, I. M. (1995). Adapting to unknown smoothness via wavelet shrinkage. Journal of the American Statistical Association 90 1200-1224.

Donoho, D.L., Johnstone, I.M., Kerkyacharian, G. and Picard, D. (1996). Density estimation by wavelet thresholding. The Annals of Statistics 24 508-539.

Hall, P. and Zhou, X.H. (2003). Nonparametric estimation of component distributions in a multivariate mixture. The Annals of Statistics 31 201-224.

Härdle, W., Kerkyacharian, G., Picard, D. and Tsybakov, A. (1998). Wavelet, Approximation and Statistical Applications. Lectures Notes in Statistics, 129, Springer Verlag, New York.

LEPSKI, O. (1990). On a problem of adaptive estimation in Gaussian white noise. Th. Prob. 35 454-466.

LePsKI, O. (1991). Asymptotically minimax adaptive estimation I : upper bounds. Optimally adaptive estimates. Th. Prob. Appl. 36 682-697.

LEPSKI, O. (1992). Asymptotically minimax adaptive estimation II : statistical models without optimal adaptation. Adaptive estimators. Th. Prob. Appl. 37 433-448.

LePski, O. And Spokoiny V.G. (1995). Local adaptation to inhomogeneous smoothness : resolution level. Math. Methods Statist. 4 239-258

MAiboroda, R. E. (1996). Estimators of components of a mixture with varying concentrations. Ukrain. Mat. Zh. 48 562-566. 
Mallat, S. (2009). A wavelet tour of signal processing. Elsevier/ Academic Press, Amsterdam, third edition. The sparse way, With contributions from Gabriel Peyré.

Meyer, Y. (1990). Ondelettes et Opérateurs. Hermann, Paris.

Petrov, V. V. (1995). Limit Theorems of Probability Theory: Sequences of Independent Random Variables. Oxford: Clarendon Press.

POKHYL'KO, D. (2005). Wavelet estimators of a density constructed from observations of a mixture. Theor. Prob. and Math. Statist. 70 135-145.

Prakasa RaO, B. L. S. (1983). Nonparametric functional estimation. Academic Press, Orlando.

Prakasa RaO, B. L. S. (1996). Nonparametric estimation of the derivatives of a density by the method of wavelets. Bull. Inform. Cyb. 28 91-100.

Prakasa RAO, B. L. S. (1999). Nonparametric functional estimation: an overview. In Asymptotics, Nonparametrics and Time Series, Ed.: Subir Ghosh, 461-509, Marcel Dekker Inc., New York.

Prakasa RAO, B. L. S. (2010). Wavelet linear estimation for derivatives of a density from observations of mixtures with varying mixing proportions. Indian Journal of Pure and Applied Mathematics 41 275-291.

Rosenthal, H. P. (1970). On the subspaces of $\mathbb{L}^{p}(p \geq 2)$ spanned by sequences of independent random variables. Israel Journal of Mathematics 20 273-303.

Tsybakov, A.B. (2004). Introduction à l'estimation non-paramétrique. Springer, New York.

VARDI, Y. (1989). Multiplicative censoring, renewal processes, deconvolution and decreasing density: Nonparametric estimation. Biometrika 76 751-761.

VARDI, Y. AND ZHANG, C. H. (1992). Large sample study of empirical distributions in a random multiplicative censoring model. The Annals of Statistics 20 1022-1039. 